\title{
Necessary and sufficient conditions for boundedness of multilinear fractional integrals with rough kernels on Morrey type spaces
}

\author{
Yanlong Shi ${ }^{1}$, Zengyan $\mathrm{Si}^{2}$, Xiangxing $\mathrm{TaO}^{3 *}$ and Yafeng $\mathrm{Shi}^{4,5}$
}

\section{"Correspondence: \\ xxtao@hotmail.com \\ ${ }^{3}$ Faculty of Science, Zhejiang \\ University of Science \& Technology, Hangzhou, Zhejiang 310023, P.R. \\ China \\ Full list of author information is \\ available at the end of the article}

\begin{abstract}
In this article, we study necessary and sufficient conditions on the parameters of the boundedness on Morrey spaces and modified Morrey spaces for $T_{\Omega, \alpha}$ and $M_{\Omega, \alpha}$, which are a multilinear fractional integral and a multilinear fractional maximal operator with rough kernel, respectively. Our results extend some known results significantly.
\end{abstract}

MSC: 42B20; 42B25; 42B35

Keywords: multilinear maximal operator; multilinear fractional integrals; rough kernel; Morrey spaces; modified Morrey spaces

\section{Introduction}

Suppose that $\Omega$ is homogeneous of degree zero on $\mathbb{R}^{n}$ and $\Omega \in L^{s}\left(\mathbb{S}^{n-1}\right)$ with $1<s \leq \infty$, where $\mathbb{S}^{n-1}$ denotes the unit sphere of $\mathbb{R}^{n}$. Moreover, $m \geq 1$ will denote an integer, $\theta_{j}(j=$ $1, \ldots, m)$ will be fixed, distinct, and nonzero real numbers, and $0<\alpha<n$. We denote $\mathbf{f}=$ $\left(f_{1}, \ldots, f_{m}\right)$, then the multilinear fractional integral operator on $\mathbb{R}^{n}$ is given by the formula

$$
T_{\Omega, \alpha} \mathbf{f}(x)=\int_{\mathbb{R}^{n}} \frac{\Omega(y)}{|y|^{n-\alpha}} \prod_{j=1}^{m} f_{j}\left(x-\theta_{j} y\right) d y
$$

and the multilinear fractional maximal operator $M_{\Omega, \alpha}$ is given by

$$
M_{\Omega, \alpha} \mathbf{f}(x)=\sup _{r>0} \frac{1}{r^{n-\alpha}} \int_{|y|<r}|\Omega(y)| \prod_{j=1}^{m}\left|f_{j}\left(x-\theta_{j} y\right)\right| d y .
$$

If $\alpha=0$, then $M_{\Omega} \equiv M_{\Omega, 0}$ is the multilinear maximal operator.

When $m=1$ and $\Omega \equiv 1$, if let $\theta_{1}=1, T_{\Omega, \alpha}$ will be the Riesz potential operator $I_{\alpha}[1,2]$ given by

$$
I_{\alpha} f(x)=\int_{\mathbb{R}^{n}} \frac{f(x-y)}{|y|^{n-\alpha}} d y .
$$

\section{Springer}

(c) 2016 Shi et al. This article is distributed under the terms of the Creative Commons Attribution 4.0 International License (http://creativecommons.org/licenses/by/4.0/), which permits unrestricted use, distribution, and reproduction in any medium, provided you give appropriate credit to the original author(s) and the source, provide a link to the Creative Commons license, and indicate if changes were made. 
Spanne and Adams obtained two remarkable results on Morrey spaces (see Definition 2.1 in Section 2) for $I_{\alpha}$. Their results can be summarized as follows.

Proposition 1.1 $[3,4]$ (Spanne, but published by Peetre) Let $0<\alpha<n, 1 \leq p<n / \alpha, 0 \leq$ $\lambda<n-\alpha p, 1 / q=1 / p-\alpha / n$, and $\mu / q=\lambda / p$. Then for $p>1$, the operator $I_{\alpha}$ is bounded from $L^{p, \lambda}\left(\mathbb{R}^{n}\right)$ to $L^{q, \mu}\left(\mathbb{R}^{n}\right)$ and for $p=1, I_{\alpha}$ is bounded from $L^{1, \lambda}\left(\mathbb{R}^{n}\right)$ to $W L^{q, \mu}\left(\mathbb{R}^{n}\right)$.

Proposition 1.2 [5, 6] Let $0<\alpha<n, 1 \leq p<n / \alpha, 0 \leq \lambda<n-\alpha p$.

(i) If $p>1$, then the condition $1 / p-1 / q=\alpha /(n-\lambda)$ is necessary and sufficient for the boundedness of the operator $I_{\alpha}$ from $L^{p, \lambda}\left(\mathbb{R}^{n}\right)$ to $L^{q, \lambda}\left(\mathbb{R}^{n}\right)$.

(ii) If $p=1$, then the condition $1-1 / q=\alpha /(n-\lambda)$ is necessary and sufficient for the boundedness of the operator $I_{\alpha}$ from $L^{1, \lambda}\left(\mathbb{R}^{n}\right)$ to $W L^{q, \lambda}\left(\mathbb{R}^{n}\right)$.

If $\lambda=0$, then the statement of Propositions 1.1 and 1.2 reduces to the well-known HardyLittlewood-Sobolev inequality. On the other hand, in 2011, Guliyev et al. [6] found this inequality in modified Morrey spaces (see Definition 2.2 in Section 2) was also valid and proved the following.

Proposition 1.3 [6] Let $0<\alpha<n, 1 \leq p<n / \alpha, 0 \leq \lambda<n-\alpha p$.

(i) If $p>1$, then the condition $\alpha / n \leq 1 / p-1 / q \leq \alpha /(n-\lambda)$ is necessary and sufficient for the boundedness of the operator $I_{\alpha}$ from $\widetilde{L}^{p, \lambda}$ to $\widetilde{L}^{q, \lambda}$.

(ii) If $p=1$, then the condition $\alpha / n \leq 1-1 / q \leq \alpha /(n-\lambda)$ is necessary and sufficient for the boundedness of the operator $I_{\alpha}$ from $\widetilde{L}^{1, \lambda}$ to $W \widetilde{L}^{q, \lambda}$.

When $m \geq 2$ and $\Omega \equiv 1$, Grafakos [7] studied Lebesgue boundedness of $T_{1, \alpha}$. Recently, Gunawan [8] extended Grafakos' result to Morrey spaces and provided a multi-version for the sufficiency of conclusion (i) in Proposition 1.2.

Proposition 1.4 [8] Let $0<\alpha<n, p$ be the harmonic mean of $p_{1}, \ldots, p_{m}>1,1<p<n / \alpha$, $0 \leq \lambda<n-\alpha p, 1 / p-1 / q=\alpha /(n-\lambda)$, then the operator $T_{1, \alpha}$ is bounded from $L^{p_{1}, \lambda}\left(\mathbb{R}^{n}\right) \times$ $\cdots \times L^{p_{m}, \lambda}\left(\mathbb{R}^{n}\right)$ to $L^{q, \lambda}\left(\mathbb{R}^{n}\right)$.

When $m \geq 2$ and $\Omega \in L^{s}\left(\mathbb{S}^{n-1}\right)$, Ding and Lu [9] studied the $L^{p_{1}} \times \cdots \times L^{p_{m}}$ boundedness for $T_{\Omega, \alpha}$. After this work above, a natural question is: what properties does the operator $T_{\Omega, \alpha}$ have on Morrey and modified Morrey spaces? We give answers as follows.

Theorem 1.1 Let $0<\alpha<n, \Omega \in L^{s}\left(\mathbb{S}^{n-1}\right)$ with $1<s \leq \infty, s^{\prime}=s /(s-1)$, $p$ be the harmonic mean of $p_{1}, \ldots, p_{m}>1,0 \leq \lambda<n-\alpha p, 1 \leq p<n / \alpha$ and satisfy

$$
\frac{\lambda}{p}=\sum_{j=1}^{m} \frac{\lambda_{j}}{p_{j}} \text { for } 0 \leq \lambda_{j}<n .
$$

(i) If $p>s^{\prime}$, then the condition $1 / p-1 / q=\alpha /(n-\lambda)$ is necessary and sufficient for the boundedness of the operator $T_{\Omega, \alpha}$ from $L^{p_{1}, \lambda_{1}}\left(\mathbb{R}^{n}\right) \times \cdots \times L^{p_{m}, \lambda_{m}}\left(\mathbb{R}^{n}\right)$ to $L^{q, \lambda}\left(\mathbb{R}^{n}\right)$.

(ii) If $p=s^{\prime}$, then the condition $1 / s^{\prime}-1 / q=\alpha /(n-\lambda)$ is necessary and sufficient for the boundedness of the operator $T_{\Omega, \alpha}$ from $L^{p_{1}, \lambda_{1}}\left(\mathbb{R}^{n}\right) \times \cdots \times L^{p_{m}, \lambda_{m}}\left(\mathbb{R}^{n}\right)$ to $W L^{q, \lambda}\left(\mathbb{R}^{n}\right)$.

Moreover, similar conclusions hold for $M_{\Omega, \alpha}$. 
Theorem 1.2 Let $\alpha, \Omega, s, p_{j}, \lambda_{j}, p$, and $\lambda$ be as in Theorem 1.1.

(i) If $p>s^{\prime}$, then the condition $\alpha / n \leq 1 / p-1 / q \leq \alpha /(n-\lambda)$ is necessary and sufficient for the boundedness of the operator $T_{\Omega, \alpha}$ from $\widetilde{L}^{p_{1}, \lambda_{1}}\left(\mathbb{R}^{n}\right) \times \cdots \times \widetilde{L}^{p_{m}, \lambda_{m}}\left(\mathbb{R}^{n}\right)$ to $\widetilde{L}^{q, \lambda}\left(\mathbb{R}^{n}\right)$.

(ii) If $p=s^{\prime}$, then the condition $\alpha / n \leq 1 / s^{\prime}-1 / q \leq \alpha /(n-\lambda)$ is necessary and sufficient for the boundedness of the operator $T_{\Omega, \alpha}$ from $\widetilde{L}^{p_{1}, \lambda_{1}}\left(\mathbb{R}^{n}\right) \times \cdots \times \widetilde{L}^{p_{m}, \lambda_{m}}\left(\mathbb{R}^{n}\right)$ to

$W \widetilde{L}^{q, \lambda}\left(\mathbb{R}^{n}\right)$.

Moreover, similar estimates hold for $M_{\Omega, \alpha}$.

Remark 1.1 Note that Theorems 1.1 and 1.2 covers Propositions 1.2 and 1.3, respectively. Also, the case $\lambda=\lambda_{1}=\cdots=\lambda_{m}$ and $\Omega \equiv 1$ reduces to Proposition 1.4; the case $\lambda=\lambda_{1}=$ $\cdots=\lambda_{m}=0$ gives the result of Ding and Lu [9] on Lebesgue spaces.

We observe that, in Theorems 1.1 and 1.2, the boundedness in the limiting case $p=$ $(n-\lambda) / \alpha$ remains open. In fact, when $p=n / \alpha(i . e . \lambda=0)$, Ding and Lu [9] found $M_{\Omega, \alpha}$ is bounded from $L^{p_{1}} \times \cdots \times L^{p_{m}}$ to $L^{\infty}$, but this corresponding result for $T_{\Omega, \alpha}$ in this case does not hold. Our next goal is to extend Ding and Lu's result to the case $0 \leq \lambda<n-\alpha$, as the continuation of Theorems 1.1 and 1.2.

Theorem 1.3 Let $0<\alpha<n, 0 \leq \lambda<n-\alpha, \Omega \in L^{s}\left(\mathbb{S}^{n-1}\right)$ with $1<s \leq \infty$, p be the harmonic mean of $p_{1}, \ldots, p_{m}>1$ and satisfy (1.1).

(i) If $p=(n-\lambda) / \alpha \geq s^{\prime}$, then the operator $M_{\Omega, \alpha}$ is bounded from $L^{p_{1}, \lambda_{1}}\left(\mathbb{R}^{n}\right) \times \cdots \times L^{p_{m}, \lambda_{m}}\left(\mathbb{R}^{n}\right)$ to $L^{\infty}\left(\mathbb{R}^{n}\right)$.

(ii) If $s^{\prime} \leq(n-\lambda) / \alpha \leq p \leq n / \alpha$, then the operator $M_{\Omega, \alpha}$ is bounded from $\widetilde{L}^{p_{1}, \lambda_{1}}\left(\mathbb{R}^{n}\right) \times \cdots \times \widetilde{L}^{p_{m}, \lambda_{m}}\left(\mathbb{R}^{n}\right)$ to $L^{\infty}\left(\mathbb{R}^{n}\right)$.

Finally we shall describe the organization of this paper. In the following section, we will study the boundedness of maximal operator $M_{\Omega}$ on Morrey and modified Morrey spaces. The last section we will devote to the boundedness of $T_{\Omega, \alpha}$ and $M_{\Omega, \alpha}$ and to showing the proof of Theorems 1.1, 1.2 and 1.3.

Throughout this paper, we assume the letter $C$ always remains to denote a positive constant that may vary at each occurrence but is independent of the essential variables.

\section{Boundedness of maximal operator $M_{\boldsymbol{\Omega}}$}

In this part, we investigate the boundedness of maximal operator $M_{\Omega}$ (see Section 1) on Morrey and modified Morrey spaces defined by the following definitions.

Definition 2.1 $[3-5,10]$ Let $1 \leq p<\infty, 0 \leq \lambda \leq n$. We denote by $L^{p, \lambda}=L^{p, \lambda}\left(\mathbb{R}^{n}\right)$ the Morrey space, and by $W L^{p, \lambda}=W L^{p, \lambda}\left(\mathbb{R}^{n}\right)$ the weak Morrey space, as the set of locally integrable functions $f(x), x \in \mathbb{R}^{n}$, with the finite norms

$$
\begin{aligned}
& \|f\|_{L^{p, \lambda}\left(\mathbb{R}^{n)}\right.}=\sup _{x \in \mathbb{R}^{n}, t>0}\left(\frac{1}{t^{\lambda}} \int_{B(x, t)}|f(y)|^{p} d y\right)^{\frac{1}{p}}, \\
& \|f\|_{W L^{p, \lambda}\left(\mathbb{R}^{n}\right)}=\sup _{r>0} r \sup _{x \in \mathbb{R}^{n}, t>0}\left(\frac{1}{t^{\lambda}}|\{y \in B(x, t):|f(y)|>r\}|\right)^{\frac{1}{p}},
\end{aligned}
$$

respectively. 
Definition $2.2[6]$ Let $1 \leq p<\infty, 0 \leq \lambda \leq n,[t]_{1}=\min \{1, t\}$. We denote by $\widetilde{L}^{p, \lambda}=\widetilde{L}^{p, \lambda}\left(\mathbb{R}^{n}\right)$ the modified Morrey space, and by $W \widetilde{L}^{p, \lambda}=W \widetilde{L}^{p, \lambda}\left(\mathbb{R}^{n}\right)$ the weak modified Morrey space, as the set of locally integrable functions $f(x), x \in \mathbb{R}^{n}$, with the finite norms

$$
\begin{aligned}
& \|f\|_{\tilde{L}^{p, \lambda}\left(\mathbb{R}^{n}\right)}=\sup _{x \in \mathbb{R}^{n}, t>0}\left(\frac{1}{[t]_{1}^{\lambda}} \int_{B(x, t)}|f(y)|^{p} d y\right)^{\frac{1}{p}}, \\
& \|f\|_{W \tilde{L}^{p, \lambda}\left(\mathbb{R}^{n}\right)}=\sup _{r>0} r \sup _{x \in \mathbb{R}^{n}, t>0}\left(\frac{1}{[t]_{1}^{\lambda}}|\{y \in B(x, t):|f(y)|>r\}|\right)^{\frac{1}{p}},
\end{aligned}
$$

respectively.

It is easy to see that $L^{p, 0}\left(\mathbb{R}^{n}\right)=\widetilde{L}^{p, 0}\left(\mathbb{R}^{n}\right)=L^{p}\left(\mathbb{R}^{n}\right), W L^{p, 0}\left(\mathbb{R}^{n}\right)=W \widetilde{L}^{p, 0}\left(\mathbb{R}^{n}\right)=W L^{p}\left(\mathbb{R}^{n}\right)$. If $\lambda<0$ or $\lambda>n$, then $\widetilde{L}^{p, \lambda}\left(\mathbb{R}^{n}\right)=L^{p, \lambda}\left(\mathbb{R}^{n}\right)=\Theta$ where $\Theta$ is the set of all functions equivalent to 0 on $\mathbb{R}^{n}$. In addition, from [6], we know

$$
\widetilde{L}^{p, \lambda}\left(\mathbb{R}^{n}\right) \subset_{\succ} L^{p, \lambda}\left(\mathbb{R}^{n}\right) \cap L^{p}\left(\mathbb{R}^{n}\right), \quad \max \left\{\|f\|_{L^{p, \lambda}},\|f\|_{L^{p}}\right\} \leq\|f\|_{\tilde{L}^{p, \lambda}} .
$$

Recall the definition of $M_{\Omega}$, as a special case when $m=1, \Omega \equiv 1$ and $\theta_{1}=1, M_{\Omega}$ is the Hardy-Littlewood maximal operator $\mathcal{M}$. In 1994, Nakai [11] obtained the boundedness of $\mathcal{M}$ on Morrey spaces, later Guliyev [6] studied the operator $\mathcal{M}$ on modified Morrey spaces and get a result parallel to Nakai's result.

Lemma 2.1 [11] Let $1 \leq p<\infty$ and $0 \leq \lambda<n$. Then for $p>1, \mathcal{M}$ is bounded from $L^{p, \lambda}$ to $L^{p, \lambda}$ and for $p=1, \mathcal{M}$ is bounded from $L^{1, \lambda}$ to $W L^{1, \lambda}$.

Lemma 2.2 [6] Let $1 \leq p<\infty$ and $0 \leq \lambda<n$. Then for $p>1, \mathcal{M}$ is bounded from $\widetilde{L}^{p, \lambda}$ to $\widetilde{L}^{p, \lambda}$ and for $p=1, \mathcal{M}$ is bounded from $\widetilde{L}^{1, \lambda}$ to $W \widetilde{L}^{1, \lambda}$.

When $m \geq 2$ and $\Omega \in L^{s}\left(\mathbb{S}^{n-1}\right)$, we find $M_{\Omega}$ also has the same properties by providing the following multi-version of Lemmas 2.1 and 2.2.

Theorem 2.3 Let $\Omega \in L^{s}\left(\mathbb{S}^{n-1}\right)$ with $1<s \leq \infty, 0 \leq \lambda<n, p$ be the harmonic mean of $p_{1}, \ldots, p_{m}>1, p \geq s^{\prime}$ and satisfy (1.1).

(i) If $p>s^{\prime}$, there exists a positive constant $C$ such that

$$
\left\|M_{\Omega} \mathbf{f}\right\|_{L^{p, \lambda}} \leq C \prod_{j=1}^{m}\left\|f_{j}\right\|_{L^{p_{j}, \lambda_{j}}} .
$$

(ii) If $p=s^{\prime}$, there exists a positive constant $C$ such that

$$
\left\|M_{\Omega} \mathbf{f}\right\|_{W L}{ }_{W, \lambda} \leq C \prod_{j=1}^{m}\left\|f_{j}\right\|_{L^{p_{j}, \lambda}}
$$

Theorem 2.4 Let $\Omega \in L^{s}\left(\mathbb{S}^{n-1}\right)$ with $1<s \leq \infty, 0 \leq \lambda<n, p$ be the harmonic mean of $p_{1}, \ldots, p_{m}>1, p \geq s^{\prime}$ and satisfy (1.1). 
(i) If $p>s^{\prime}$, there exists a positive constant $C$ such that

$$
\left\|M_{\Omega} \mathbf{f}\right\|_{\tilde{L}^{p, \lambda}} \leq C \prod_{j=1}^{m}\left\|f_{j}\right\|_{\tilde{L}^{p_{j}, \lambda_{j}}} .
$$

(ii) If $p=s^{\prime}$, there exists a positive constant $C$ such that

$$
\left\|M_{\Omega} \mathbf{f}\right\|_{W \widetilde{L} p, \lambda} \leq C \prod_{j=1}^{m}\left\|f_{j}\right\|_{\widetilde{L}^{p j, \lambda},} .
$$

Here, we give only the proof of Theorem 2.4 and omit the proof of Theorem 2.3 due to the similarity.

Proof Since $\Omega \in L^{s}\left(\mathbb{S}^{n-1}\right)$ with $s>1$, Hölder's inequality yields

$$
\begin{aligned}
& \frac{1}{r^{n}} \int_{|y|<r}|\Omega(y)| \prod_{j=1}^{m}\left|f_{j}\left(x-\theta_{j} y\right)\right| d y \\
& \quad \leq \frac{1}{r^{n}}\left(\int_{|y|<r} \prod_{j=1}^{m}\left|f_{j}\left(x-\theta_{j} y\right)\right|^{s^{\prime}} d y\right)^{1 / s^{\prime}}\left(\int_{|y|<r}|\Omega(y)|^{s} d y\right)^{1 / s} \\
& \quad=\frac{1}{r^{n}}\left(\int_{|y|<r} \prod_{j=1}^{m}\left|f_{j}\left(x-\theta_{j} y\right)\right|^{s^{\prime}} d y\right)^{1 / s^{\prime}}\left(\int_{0}^{r} \int_{\mathbb{S}^{n-1}}|\Omega(\xi)|^{s} z^{n-1} d \xi d z\right)^{1 / s} \\
& \quad=C\left(\frac{1}{r^{n}} \int_{|y|<r} \prod_{j=1}^{m}\left|f_{j}\left(x-\theta_{j} y\right)\right|^{s^{\prime}} d y\right)^{1 / s^{\prime}} \\
& \quad \leq C \prod_{j=1}^{m}\left(\frac{1}{r^{n}} \int_{|y|<r}\left|f_{j}\left(x-\theta_{j} y\right)\right|^{s^{\prime} p_{j} / p} d y\right)^{p / s^{\prime} p_{j}} \\
& \quad \leq C \prod_{j=1}^{m}\left[\mathcal{M}\left(f_{j}^{s^{\prime} p_{j} / p}\right)(x)\right]^{p / s^{\prime} p_{j}},
\end{aligned}
$$

which implies a pointwise estimate

$$
M_{\Omega} \mathbf{f}(x) \leq C \prod_{j=1}^{m}\left[\mathcal{M}\left(f_{j}^{s^{\prime} p_{j} / p}\right)(x)\right]^{p / s^{\prime} p_{j}}
$$

(i) If $p>s^{\prime}$, by (2.1) and the Hölder inequality, we get

$$
\begin{aligned}
\frac{1}{[t]_{1}^{\lambda}} \int_{B(x, t)}\left|M_{\Omega} \mathbf{f}(y)\right|^{p} d y & \leq C \frac{1}{[t]_{1}^{\lambda}} \int_{B(x, t)} \prod_{j=1}^{m}\left[\mathcal{M}\left(f_{j}^{s^{\prime} p_{j} / p}\right)(y)\right]^{p^{2} / s^{\prime} p_{j}} d y \\
& \leq C \prod_{j=1}^{m}\left(\frac{1}{[t]_{1}^{\lambda_{j}}} \int_{B(x, t)}\left[\mathcal{M}\left(f_{j}^{s^{\prime} p_{j} / p}\right)(y)\right]^{p / s^{\prime}} d y\right)^{p / p_{j}}
\end{aligned}
$$

for all $x \in \mathbb{R}^{n}$ and $t>0$. 
Taking the $p$ th root of both sides and applying Lemma 2.2 with $p / s^{\prime}>1$ and the fact $f_{j}^{s^{\prime} p_{j} / p} \in L^{p / s^{\prime}, \lambda_{j}}$, we get

$$
\begin{aligned}
\left\|M_{\Omega} \mathbf{f}\right\|_{\tilde{L}^{p, \lambda}} & =\sup _{x \in \mathbb{R}^{n}, t>0}\left(\frac{1}{[t]_{1}^{\lambda}} \int_{B(x, t)}\left|M_{\Omega} \mathbf{f}(y)\right|^{p} d y\right)^{1 / p} \\
& \leq C \prod_{j=1}^{m} \sup _{x \in \mathbb{R}^{n}, t>0}\left(\frac{1}{[t]_{1}^{\lambda_{j}}} \int_{B(x, t)}\left[\mathcal{M}\left(f_{j}^{s^{\prime} p_{j} / p}\right)(y)\right]^{p / s^{\prime}} d y\right)^{1 / p_{j}} \\
& =C \prod_{j=1}^{m}\left\|\mathcal{M}\left(f_{j}^{s^{\prime} p_{j} / p}\right)\right\|_{\widetilde{L}^{p / s^{\prime}, \lambda_{j}}}^{p / p_{j}} \\
& \leq C \prod_{j=1}^{m}\left\|f_{j}^{s^{\prime} p_{j} / p}\right\|_{\widetilde{L}^{p / s^{\prime}, \lambda_{j}}}^{p / s^{\prime} p_{j}} \\
& =C \prod_{j=1}^{m}\left\|f_{j}\right\|_{\widetilde{L}^{p, \lambda_{j}},}
\end{aligned}
$$

which is the desired inequality.

(ii) If $p=s^{\prime}$, for any $\beta>0$, let $\varepsilon_{0}=\beta, \varepsilon_{m}=1$ and $\varepsilon_{1}, \varepsilon_{2}, \ldots, \varepsilon_{m-1}>0$ be arbitrary which will be chosen later. From the pointwise estimate (2.1), we get

$$
\left\{y \in B(x, t):\left|M_{\Omega} \mathbf{f}(y)\right|>\beta\right\} \subset \bigcup_{j=1}^{m}\left\{y \in B(x, t):\left[\mathcal{M}\left(f_{j}^{s^{\prime} p_{j} / p}\right)(y)\right]^{p / s^{\prime} p_{j}}>\frac{\varepsilon_{j-1}}{[t]_{1}^{\left(\lambda-\lambda_{j}\right) / p_{j}} \varepsilon_{j}}\right\} .
$$

Let us now take $\varepsilon_{1}, \varepsilon_{2}, \ldots, \varepsilon_{m-1}>0$ such that

$$
\frac{\varepsilon_{j}}{\varepsilon_{j-1}}=\frac{\left[\prod_{j=1}^{m}\left\|f_{j}\right\|_{\tilde{L}_{j}^{p_{j}, \lambda_{j}}}\right]^{s^{\prime} / p_{j}}}{\beta^{s^{\prime} / p_{j}}\left\|f_{j}\right\|_{\tilde{L}^{p_{j}, \lambda_{j}}}}, \quad j=1,2, \ldots, m .
$$

Then, applying Lemma 2.2 with $p / s^{\prime}=1$ and the fact $f_{j}^{p_{j}} \in L^{1, \lambda_{j}}$, we get

$$
\begin{aligned}
\mid\{y & \left.\in B(x, t):\left|M_{\Omega} \mathbf{f}(y)\right|>\beta\right\} \mid \\
& \leq C \sum_{j=1}^{m}\left|\left\{y \in B(x, t): \mathcal{M}\left(f_{j}^{p_{j}}\right)(y)>\left(\frac{\varepsilon_{j-1}}{[t]_{1}^{\left(\lambda-\lambda_{j}\right) / p_{j}} \varepsilon_{j}}\right)^{p_{j}}\right\}\right| \\
& \leq C \sum_{j=1}^{m}[t]_{1}^{\lambda_{j}}\left(\frac{[t]_{1}^{\left(\lambda-\lambda_{j}\right) / p_{j}} \varepsilon_{j}}{\varepsilon_{j-1}}\right)^{p_{j}}\left\|f_{j}^{p_{j}}\right\|_{\widetilde{L}^{1, \lambda_{j}}} \\
& =C \sum_{j=1}^{m}[t]_{1}^{\lambda}\left(\frac{\varepsilon_{j}}{\varepsilon_{j-1}}\right)^{p_{j}}\left\|f_{j}\right\|_{\widetilde{L}^{p_{j}, \lambda_{j}}}^{p_{j}} \\
& =C \sum_{j=1}^{m}[t]_{1}^{\lambda}\left[\left(\frac{\varepsilon_{j}}{\varepsilon_{j-1}}\right)\left\|f_{j}\right\|_{\tilde{L}^{p_{j}, \lambda_{j}}}\right]^{p_{j}} \\
& =C \sum_{j=1}^{m}[t]_{1}^{\lambda}\left(\frac{1}{\beta} \prod_{j=1}^{m}\left\|f_{j}\right\|_{\tilde{L}^{p_{j}, \lambda_{j}}}\right)^{s^{\prime}} \\
& =C[t]_{1}^{\lambda}\left(\frac{1}{\beta} \prod_{j=1}^{m}\left\|f_{j}\right\|_{\tilde{L}^{p_{j}, \lambda_{j}}}\right)^{p} .
\end{aligned}
$$


Hence, we obtain the following inequality:

$$
\begin{aligned}
\left\|M_{\Omega} \mathbf{f}\right\|_{W \widetilde{L} p, \lambda} & =\sup _{\beta>0} \beta \sup _{x \in \mathbb{R}^{n}, t>0}\left(\frac{1}{[t]_{1}^{\lambda}}\left|\left\{y \in B(x, t):\left|M_{\Omega} \mathbf{f}(y)\right|>\beta\right\}\right|\right)^{\frac{1}{p}} \\
& \leq C \prod_{j=1}^{m}\left\|f_{j}\right\|_{\widetilde{L}^{p_{j}, \lambda_{j}} .}
\end{aligned}
$$

This is the conclusion (ii) of Theorem 2.4.

\section{Boundedness of $T_{\Omega, \alpha}$ and $M_{\Omega, \alpha}$}

The present section consists of two parts which are about the bounded estimates on Morrey and modified spaces for the multilinear fractional integral operator $T_{\Omega, \alpha}$ and the multilinear fractional maximal operator $M_{\Omega, \alpha}$, respectively.

\subsection{Boundedness on Morrey spaces}

In this part, we will prove Theorem 1.1. Let us begin with a requisite Hedberg's type estimates, which plays a key role in proving Theorem 1.1.

Lemma 3.1 Let $0<\alpha<n, \Omega \in L^{s}\left(\mathbb{S}^{n-1}\right)$ with $1<s \leq \infty$, $p$ be the harmonic mean of $p_{1}, \ldots, p_{m}>1,0 \leq \lambda<n-\alpha p, s^{\prime} \leq p<n / \alpha$ and satisfy (1.1), then there exists a positive constant $C$ such that

$$
\left|T_{\Omega, \alpha} \mathbf{f}(x)\right| \leq C\left[M_{\Omega} \mathbf{f}(x)\right]^{1-p \alpha /(n-\lambda)} \prod_{j=1}^{m}\left\|f_{j}\right\|_{L^{p_{j}, \lambda_{j}}}^{p \alpha /(n-\lambda)}
$$

Proof For any $\delta>0$, we split the integral into two parts:

$$
T_{\Omega, \alpha} \mathbf{f}(x)=\left(\int_{|y|<\delta}+\int_{|y| \geq \delta}\right) \frac{\Omega(y)}{|y|^{n-\alpha}} \prod_{j=1}^{m} f_{j}\left(x-\theta_{j} y\right) d y=: A(x, \delta)+S(x, \delta) .
$$

For $A(x, \delta)$, we have

$$
\begin{aligned}
|A(x, \delta)| & \leq \int_{|y|<\delta} \frac{|\Omega(y)|}{|y|^{n-\alpha}} \prod_{j=1}^{m}\left|f_{j}\left(x-\theta_{j} y\right)\right| d y \\
& \leq \sum_{i=0}^{\infty} \int_{2^{-i-1} \delta \leq|y|<2^{-i} \delta} \frac{|\Omega(y)|}{|y|^{n-\alpha}} \prod_{j=1}^{m}\left|f_{j}\left(x-\theta_{j} y\right)\right| d y \\
& \leq \sum_{i=0}^{\infty}\left(2^{-i-1} \delta\right)^{\alpha-n} \int_{|y|<2^{-i} \delta}|\Omega(y)| \prod_{j=1}^{m}\left|f_{j}\left(x-\theta_{j} y\right)\right| d y \\
& \leq \sum_{i=0}^{\infty}\left(2^{-i-1} \delta\right)^{\alpha-n}\left(2^{-i} \delta\right)^{n} M_{\Omega} \mathbf{f}(x) \\
& \leq 2^{n-\alpha} \delta^{\alpha} M_{\Omega} \mathbf{f}(x) \sum_{i=0}^{\infty} 2^{-i \alpha} \\
& \leq C \delta^{\alpha} M_{\Omega} \mathbf{f}(x) .
\end{aligned}
$$


Recalling the conditions of Lemma 3.1, we can see $s^{\prime} \leq p<(n-\lambda) / \alpha$, which implies $\alpha<(n-\lambda) / p \leq(n-\lambda) / s^{\prime}$, then we get

$$
n-\alpha s^{\prime}>n-(n-\lambda) s^{\prime} / p \geq n-(n-\lambda)=\lambda .
$$

In order to estimate $S(x, \delta)$, we choose a real number $\sigma$ such that

$$
n-\alpha s^{\prime}>\sigma>n-(n-\lambda) s^{\prime} / p \geq \lambda .
$$

One can then see from the choice of $\sigma$ that

$$
n-\left(n-\alpha-\sigma / s^{\prime}\right) s<0
$$

and

$$
(n-\sigma) / s^{\prime}-(n-\lambda) / p<0 .
$$

Then, using the Hölder inequality, we obtain

$$
\begin{aligned}
|S(x, \delta)| & \leq \int_{|y| \geq \delta} \frac{|\Omega(y)|}{|y|^{n-\alpha-\sigma / s^{\prime}}} \frac{1}{|y|^{\sigma / s^{\prime}}} \prod_{j=1}^{m}\left|f_{j}\left(x-\theta_{j} y\right)\right| d y \\
& \leq\left(\int_{|y| \geq \delta} \frac{|\Omega(y)|^{s}}{|y|^{\left(n-\alpha-\sigma / s^{\prime}\right) s}} d y\right)^{1 / s}\left(\int_{|y| \geq \delta} \frac{1}{|y|^{\sigma}} \prod_{j=1}^{m}\left|f_{j}\left(x-\theta_{j} y\right)\right|^{s^{\prime}} d y\right)^{1 / s^{\prime}} \\
& =: E_{\sigma}(\delta) \times F_{\sigma}(x, \delta) .
\end{aligned}
$$

For $E_{\sigma}(\delta)$, by the fact (3.1), we obtain

$$
E_{\sigma}(\delta)=\left(\int_{\delta}^{\infty} \int_{\mathbb{S}^{n-1}}|\Omega(\xi)|^{s} r^{n-\left(n-\alpha-\sigma / s^{\prime}\right) s-1} d \xi d r\right)^{1 / s}=C \delta^{\alpha-(n-\sigma) / s^{\prime}}
$$

For $F_{\sigma}(x, \delta)$, we have

$$
\begin{aligned}
F_{\sigma}(x, \delta) & \leq\left(\sum_{i=0}^{\infty} \int_{2^{i} \delta \leq|y|<2^{i+1} \delta} \frac{1}{|y|^{\sigma}} \prod_{j=1}^{m}\left|f_{j}\left(x-\theta_{j} y\right)\right|^{s^{\prime}} d y\right)^{1 / s^{\prime}} \\
& \leq \sum_{i=0}^{\infty}\left(2^{i} \delta\right)^{-\sigma / s^{\prime}}\left(\int_{|y|<2^{i+1} \delta} \prod_{j=1}^{m}\left|f_{j}\left(x-\theta_{j} y\right)\right|^{s^{\prime}} d y\right)^{1 / s^{\prime}} .
\end{aligned}
$$

If $p>s^{\prime}$, applying Hölder's inequality and the fact (3.2), we have

$$
\begin{aligned}
F_{\sigma}(x, \delta) & \leq \sum_{i=0}^{\infty}\left(2^{i} \delta\right)^{-\sigma / s^{\prime}}\left(\int_{|y|<2^{i+1} \delta} d y\right)^{1 / s^{\prime}-1 / p}\left(\int_{|y|<2^{i+1} \delta} \prod_{j=1}^{m}\left|f_{j}\left(x-\theta_{j} y\right)\right|^{p} d y\right)^{1 / p} \\
& \leq C \sum_{i=0}^{\infty}\left(2^{i} \delta\right)^{(n-\sigma) / s^{\prime}-n / p}\left(\int_{|y|<2^{i+1} \delta} \prod_{j=1}^{m}\left|f_{j}\left(x-\theta_{j} y\right)\right|^{p} d y\right)^{1 / p}
\end{aligned}
$$


Shr et al. Journal of Inequalities and Applications ( 2016) 2016:43

Page 9 of 19

$$
\begin{aligned}
& \leq C \sum_{i=0}^{\infty}\left(2^{i} \delta\right)^{(n-\sigma) / s^{\prime}-(n-\lambda) / p}\left(\frac{1}{\left(2^{i+1} \delta\right)^{\lambda}} \int_{|y|<2^{i+1} \delta} \prod_{j=1}^{m}\left|f_{j}\left(x-\theta_{j} y\right)\right|^{p} d y\right)^{1 / p} \\
& \leq C \sum_{i=0}^{\infty}\left(2^{i} \delta\right)^{(n-\sigma) / s^{\prime}-(n-\lambda) / p} \prod_{j=1}^{m}\left(\frac{1}{\left(2^{i+1} \delta\right)^{\lambda_{j}}} \int_{|y|<2^{i+1} \delta}\left|f_{j}\left(x-\theta_{j} y\right)\right|^{p_{j}} d y\right)^{1 / p_{j}} \\
& \leq C \sum_{i=0}^{\infty}\left(2^{i} \delta\right)^{(n-\sigma) / s^{\prime}-(n-\lambda) / p} \prod_{j=1}^{m}\left\|f_{j}\right\|_{L^{p_{j}, \lambda_{j}}} \\
& \leq C \delta^{(n-\sigma) / s^{\prime}-(n-\lambda) / p} \prod_{j=1}^{m}\left\|f_{j}\right\|_{L^{p_{j}, \lambda_{j}}} .
\end{aligned}
$$

If $p=s^{\prime}$, using the Hölder inequality and the fact $\lambda<\sigma$, we get

$$
\begin{aligned}
F_{\sigma}(x, \delta) & \leq \sum_{i=0}^{\infty}\left(2^{i} \delta\right)^{-\sigma / s^{\prime}}\left(\int_{|y|<2^{i+1} \delta} \prod_{j=1}^{m}\left|f_{j}\left(x-\theta_{j} y\right)\right|^{s^{\prime}} d y\right)^{1 / s^{\prime}} \\
& \leq C \sum_{i=0}^{\infty}\left(2^{i} \delta\right)^{-\sigma / s^{\prime}+\lambda / s^{\prime}}\left(\frac{1}{\left(2^{i+1} \delta\right)^{\lambda}} \int_{|y|<2^{i+1} \delta} \prod_{j=1}^{m}\left|f_{j}\left(x-\theta_{j} y\right)\right|^{s^{\prime}} d y\right)^{1 / s^{\prime}} \\
& \leq C \sum_{i=0}^{\infty}\left(2^{i} \delta\right)^{(\lambda-\sigma) / s^{\prime}} \prod_{j=1}^{m}\left(\frac{1}{\left(2^{i+1} \delta\right)^{\lambda_{j}}} \int_{|y|<2^{i+1} \delta}\left|f_{j}\left(x-\theta_{j} y\right)\right|^{p_{j}} d y\right)^{1 / p_{j}} \\
& \leq C \sum_{i=0}^{\infty}\left(2^{i} \delta\right)^{(\lambda-\sigma) / s^{\prime}} \prod_{j=1}^{m}\left\|f_{j}\right\|_{L^{p_{j}, \lambda_{j}}} \\
& \leq C \delta^{(\lambda-\sigma) / s^{\prime}} \prod_{j=1}^{m}\left\|f_{j}\right\|_{L^{p_{j}, \lambda_{j}}} \\
& =C \delta^{(n-\sigma) / s^{\prime}-(n-\lambda) / p} \prod_{j=1}^{m}\left\|f_{j}\right\|_{L^{p_{j}, \lambda_{j}} .}
\end{aligned}
$$

Hence, for every $p \geq s^{\prime}$, we have

$$
|S(x, \delta)| \leq C \delta^{\alpha-(n-\lambda) / p} \prod_{j=1}^{m}\left\|f_{j}\right\|_{L^{p_{j}, \lambda_{j}}}
$$

Thus

$$
\left|T_{\Omega, \alpha} \mathbf{f}(x)\right| \leq C\left(\delta^{\alpha} M_{\Omega} \mathbf{f}(x)+\delta^{\alpha-(n-\lambda) / p} \prod_{j=1}^{m}\left\|f_{j}\right\|_{L^{p_{j}, \lambda_{j}}}\right), \quad \delta>0 .
$$

Now take

$$
\delta=\left[\left(M_{\Omega} \mathbf{f}(x)\right)^{-1} \prod_{j=1}^{m}\left\|f_{j}\right\|_{L^{p_{j}, \lambda_{j}}}\right]^{p /(n-\lambda)}
$$

and then we get the conclusion of Lemma 3.1. 
Now we are ready to prove Theorem 1.1.

Proof of Theorem 1.1 First of all, we will devote our efforts to the proof of (i).

Sufficiency. By Lemma 3.1 and the conclusion (i) of Theorem 2.3, we have

$$
\begin{aligned}
\left(\frac{1}{t^{\lambda}} \int_{B(x, t)}\left|T_{\Omega, \alpha} \mathbf{f}(y)\right|^{q} d y\right)^{1 / q} & \leq C \prod_{j=1}^{m}\left\|f_{j}\right\|_{L^{p_{j}, \lambda_{j}}}^{1-q}\left(\frac{1}{t^{\lambda}} \int_{B(x, t)}\left(M_{\Omega} \mathbf{f}(y)\right)^{p} d y\right)^{1 / q} \\
& \leq C \prod_{j=1}^{m}\left\|f_{j}\right\|_{L^{p_{j}, \lambda_{j}}}^{1-p / q} \prod_{j=1}^{m}\left\|f_{j}\right\|_{L^{p_{j}, \lambda_{j}}}^{p / q} \\
& \leq C \prod_{j=1}^{m}\left\|f_{j}\right\|_{L^{p_{j}, \lambda_{j}}} .
\end{aligned}
$$

Taking the supremum for $x \in \mathbb{R}^{n}$ and $t>0$, we will get the desired conclusion.

Necessity. Suppose that $T_{\Omega, \alpha}$ is bounded from $L^{p_{1}, \lambda_{1}} \times \cdots \times L^{p_{m}, \lambda_{m}}$ to $L^{q, \lambda}$. Define $\mathbf{f}_{\epsilon}(x)=$ $\left(f_{1}(\epsilon x), \ldots, f_{m}(\epsilon x)\right)$ for $\epsilon>0$. Then it is easy to show that

$$
T_{\Omega, \alpha} \mathbf{f}_{\epsilon}(y)=\epsilon^{-\alpha} T_{\Omega, \alpha} \mathbf{f}(\epsilon y) .
$$

Thus

$$
\begin{aligned}
\left\|T_{\Omega, \alpha} \mathbf{f}_{\epsilon}\right\|_{L^{q, \lambda}} & =\epsilon^{-\alpha} \sup _{x \in \mathbb{R}^{n}, t>0}\left(\frac{1}{t^{\lambda}} \int_{B(x, t)}\left|T_{\Omega, \alpha} \mathbf{f}(\epsilon y)\right|^{q} d y\right)^{1 / q} \\
& =\epsilon^{-\alpha-n / q} \sup _{x \in \mathbb{R}^{n}, t>0}\left(\frac{1}{t^{\lambda}} \int_{B(\epsilon x, \epsilon t)}\left|T_{\Omega, \alpha} \mathbf{f}(y)\right|^{q} d y\right)^{1 / q} \\
& =\epsilon^{-\alpha-n / q+\lambda / q} \sup _{x \in \mathbb{R}^{n}, t>0}\left(\frac{1}{(\epsilon t)^{\lambda}} \int_{B(\epsilon x, \epsilon t)}\left|T_{\Omega, \alpha} \mathbf{f}(y)\right|^{q} d y\right)^{1 / q} \\
& =\epsilon^{-\alpha-(n-\lambda) / q}\left\|T_{\Omega, \alpha} \mathbf{f}\right\|_{L^{q, \lambda}}
\end{aligned}
$$

Since $T_{\Omega, \alpha}$ is bounded from $L^{p_{1}, \lambda_{1}} \times \cdots \times L^{p_{m}, \lambda_{m}}$ to $L^{q, \lambda}$, we have

$$
\begin{aligned}
\left\|T_{\Omega, \alpha} \mathbf{f}\right\|_{L^{q, \lambda}} & =\epsilon^{\alpha+(n-\lambda) / q}\left\|T_{\Omega, \alpha} \mathbf{f}_{\epsilon}\right\|_{L^{q, \lambda}} \\
& \leq C \epsilon^{\alpha+(n-\lambda) / q} \prod_{j=1}^{m}\left\|f_{j}(\epsilon \cdot)\right\|_{L^{p_{j}, \lambda j}} \\
& =C \epsilon^{\alpha+(n-\lambda) / q} \prod_{j=1}^{m} \sup _{x \in \mathbb{R}^{n}, t>0}\left(\frac{1}{t^{\lambda_{j}}} \int_{B(x, t)}\left|f_{j}(\epsilon y)\right|^{p_{j}} d y\right)^{1 / p_{j}} \\
& =C \epsilon^{\alpha+(n-\lambda) / q} \prod_{j=1}^{m} \epsilon^{-n / p_{j}} \sup _{x \in \mathbb{R}^{n}, t>0}\left(\frac{1}{t^{\lambda_{j}}} \int_{B(\epsilon x, \epsilon t)}\left|f_{j}(y)\right|^{p_{j}} d y\right)^{1 / p_{j}} \\
& =C \epsilon^{\alpha+(n-\lambda) / q} \prod_{j=1}^{m} \epsilon^{\left(\lambda_{j}-n\right) / p_{j}} \sup _{x \in \mathbb{R}^{n}, t>0}\left(\frac{1}{(\epsilon t)^{\lambda_{j}}} \int_{B(\epsilon x, \epsilon t)}\left|f_{j}(y)\right|^{p_{j}} d y\right)^{1 / p_{j}} \\
& =C \epsilon^{\alpha+(n-\lambda) / q-(n-\lambda) / p} \prod_{j=1}^{m}\left\|f_{j}\right\|_{L^{p_{j}, \lambda_{j}},}
\end{aligned}
$$

where $C$ is independent of $\epsilon$. 
If $1 / p<1 / q+\alpha /(n-\lambda)$, then for all $\mathbf{f} \in L^{p_{1}, \lambda_{1}} \times \cdots \times L^{p_{m}, \lambda_{m}}$, we have $\left\|T_{\Omega, \alpha} \mathbf{f}\right\|_{L^{q, \lambda}}=0$ as $\epsilon \rightarrow 0$.

If $1 / p>1 / q+\alpha /(n-\lambda)$, then for all $\mathbf{f} \in L^{p_{1}, \lambda_{1}} \times \cdots \times L^{p_{m}, \lambda_{m}}$, we have $\left\|T_{\Omega, \alpha} \mathbf{f}\right\|_{L^{q, \lambda}}=0$ as $\epsilon \rightarrow \infty$.

Therefore we get $1 / p=1 / q+\alpha /(n-\lambda)$.

We proceed to prove (ii). Sufficiency. For any $\beta>0$, applying Lemma 3.1 and the conclusion (ii) of Theorem 2.3, we get

$$
\begin{aligned}
& \left\|T_{\Omega, \alpha} \mathbf{f}\right\|_{W L^{q, \lambda}} \\
& =\sup _{\beta>0} \beta \sup _{x \in \mathbb{R}^{n}, t>0}\left(\frac{1}{t^{\lambda}}\left|\left\{y \in B(x, t):\left|T_{\Omega} \mathbf{f}(y)\right|>\beta\right\}\right|\right)^{1 / q} \\
& \leq \sup _{\beta>0} \beta \sup _{x \in \mathbb{R}^{n}, t>0}\left(\frac{1}{t^{\lambda}}\left|\left\{y \in B(x, t):\left(C M_{\Omega} \mathbf{f}(y)\right)^{s^{\prime} / q} \prod_{j=1}^{m}\left\|f_{j}\right\|_{L^{p_{j}, \lambda_{j}}}^{1-s^{\prime} / q}>\beta\right\}\right|\right)^{1 / q} \\
& \leq \sup _{\beta>0} \beta \sup _{x \in \mathbb{R}^{n}, t>0}\left[\frac{1}{t^{\lambda}}\left|\left\{y \in B(x, t): M_{\Omega} \mathbf{f}(y)>\left(\frac{\beta}{C \prod_{j=1}^{m}\left\|f_{j}\right\|_{L^{p_{j}, \lambda_{j}}}^{1-s^{\prime} / q}}\right)^{q / s^{\prime}}\right\}\right|\right]^{1 / q} \\
& \leq \sup _{\beta>0} \beta \sup _{x \in \mathbb{R}^{n}, t>0}\left[\frac{1}{t^{\lambda}}\left|\left\{y \in B(x, t): M_{\Omega} \mathbf{f}(y)>\left(\frac{\beta}{C \prod_{j=1}^{m}\left\|f_{j}\right\|_{L^{p_{j}, \lambda_{j}}}^{1-s^{\prime}}}\right)^{q / s^{\prime}}\right\}\right|\right]^{\frac{1}{s^{\prime}} \times \frac{s^{\prime}}{q}} \\
& \leq C \sup _{\beta>0} \beta\left[\left(\frac{\prod_{j=1}^{m}\left\|f_{j}\right\|_{L^{p_{j}, \lambda_{j}}}^{1-s^{\prime} / q}}{\beta}\right)^{q / s^{\prime}} \prod_{j=1}^{m}\left\|f_{j}\right\|_{L^{p_{j}, \lambda_{j}}}\right]^{s^{\prime} / q} \\
& \leq C \sup _{\beta>0} \beta\left[\frac{\prod_{j=1}^{m}\left\|f_{j}\right\|_{L^{p_{j}, \lambda_{j}}}^{1-s^{\prime} / q}}{\beta} \prod_{j=1}^{m}\left\|f_{j}\right\|_{L^{p_{j}, \lambda_{j}}}^{s^{\prime} / q}\right] \\
& \leq C \prod_{j=1}^{m}\left\|f_{j}\right\|_{L^{p_{j}, \lambda_{j}}} .
\end{aligned}
$$

Thus, we complete the sufficiency of (ii).

Necessity. Let $T_{\Omega, \alpha}$ be bounded from $L^{p_{1}, \lambda_{1}} \times \cdots \times L^{p_{m}, \lambda_{m}}$ to $W L^{q, \lambda}$. Because we have (3.3) for $\mathbf{f}_{\epsilon}(x)=\left(f_{1}(\epsilon x), \ldots, f_{m}(\epsilon x)\right)$ with $\epsilon>0$, then we obtain

$$
\begin{aligned}
\left\|T_{\Omega, \alpha} \mathbf{f}_{\epsilon}\right\|_{W L q, \lambda} & =\sup _{r>0} r \sup _{x \in \mathbb{R}^{n}, t>0}\left(\frac{1}{t^{\lambda}} \int_{\left\{y \in B(x, t):\left|T_{\Omega, \alpha} \mathbf{f}_{\epsilon}(y)\right|>r\right\}} d y\right)^{\frac{1}{q}} \\
& =\sup _{r>0} r \sup _{x \in \mathbb{R}^{n}, t>0}\left(\frac{1}{t^{\lambda}} \int_{\left\{y \in B(x, t):\left|T_{\Omega, \alpha} \mathbf{f}(\epsilon y)\right|>r \epsilon^{\alpha}\right\}} d y\right)^{\frac{1}{q}} \\
& =\epsilon^{-n / q} \sup _{r>0} r \sup _{x \in \mathbb{R}^{n}, t>0}\left(\frac{1}{t^{\lambda}} \int_{\left\{y \in B(\epsilon x, \epsilon t):\left|T_{\Omega, \alpha} \mathbf{f}(y)\right|>r \epsilon^{\alpha}\right\}} d y\right)^{\frac{1}{q}} \\
& =\epsilon^{-\alpha-n / q+\lambda / q} \sup _{r>0} r \epsilon^{\alpha} \sup _{x \in \mathbb{R}^{n}, t>0}\left(\frac{1}{(\epsilon t)^{\lambda}} \int_{\left\{y \in B(\epsilon x, \epsilon t):\left|T_{\Omega, \alpha} \mathbf{f}(y)\right|>r \epsilon^{\alpha}\right\}} d y\right)^{\frac{1}{q}} \\
& =\epsilon^{-\alpha-(n-\lambda) / q}\left\|T_{\Omega, \alpha} \mathbf{f}\right\|_{W L^{q, \lambda} .}
\end{aligned}
$$


Since $T_{\Omega, \alpha}$ is bounded from $L^{p_{1}, \lambda_{1}} \times \cdots \times L^{p_{m}, \lambda_{m}}$ to $W L^{q, \lambda}$, we have

$$
\begin{aligned}
\left\|T_{\Omega, \alpha} \mathbf{f}\right\|_{W L} q, \lambda & =\epsilon^{\alpha+(n-\lambda) / q}\left\|T_{\Omega, \alpha} \mathbf{f}_{\epsilon}\right\|_{W L^{q, \lambda}} \leq C \epsilon^{\alpha+(n-\lambda) / q} \prod_{j=1}^{m}\left\|f_{j}(\epsilon \cdot)\right\|_{L^{p_{j}, \lambda_{j}}} \\
& \leq C \epsilon^{\alpha+(n-\lambda) / q-(n-\lambda) / p} \prod_{j=1}^{m}\left\|f_{j}\right\|_{L^{p_{j}, \lambda_{j}}},
\end{aligned}
$$

where $C$ is independent of $\epsilon$.

If $1 / p<1 / q+\alpha /(n-\lambda)$, then for all $\mathbf{f} \in L^{p_{1}, \lambda} \times \cdots \times L^{p_{m}, \lambda}$, we have $\left\|T_{\Omega, \alpha} \mathbf{f}\right\|_{W L^{q, \lambda}}=0$ as $\epsilon \rightarrow 0$.

If $1 / p>1 / q+\alpha /(n-\lambda)$, then for all $\mathbf{f} \in L^{p_{1}, \lambda} \times \cdots \times L^{p_{m}, \lambda}$, we have $\left\|T_{\Omega, \alpha} \mathbf{f}\right\|_{W L^{q, \lambda}}=0$ as $\epsilon \rightarrow \infty$.

Consequently, we get $1 / p=1 / q+\alpha /(n-\lambda)$.

Next, we prove conclusions (i) and (ii) hold for $M_{\Omega, \alpha}$. By the same arguments as above we get the necessity part and the sufficiency part follows from the conclusion of $T_{\Omega, \alpha}$ and the following lemma.

Lemma 3.2 [9] Suppose that $0<\alpha<n, \Omega \in L^{s}\left(\mathbb{S}^{n-1}\right)$ with $1<s \leq \infty$. Then

$$
M_{\Omega, \alpha}(\mathbf{f})(x) \leq C_{\alpha, n} T_{|\Omega|, \alpha}(|\mathbf{f}|)(x)
$$

where $|\mathbf{f}|=\left(\left|f_{1}\right|, \ldots,\left|f_{m}\right|\right)$.

Then the proof of Theorem 1.1 is completed.

As an application of Theorem 1.1, we get Spanne type estimates, which can be seen a multi-version of Proposition 1.1.

Corollary 3.1 Let $\alpha, \Omega, s, p_{j}, \lambda, p$, and $\lambda$ be as in Theorem $1.1,1 / q=1 / p-\alpha / n, \mu / q=\lambda / p$.

(i) If $p>s^{\prime}$, then $T_{\Omega, \alpha}$ is bounded from $L^{p_{1}, \lambda_{1}}\left(\mathbb{R}^{n}\right) \times \cdots \times L^{p_{m}, \lambda_{m}}\left(\mathbb{R}^{n}\right)$ to $L^{q, \mu}\left(\mathbb{R}^{n}\right)$.

(ii) If $p=s^{\prime}$, then $T_{\Omega, \alpha}$ is bounded from $L^{p_{1}, \lambda_{1}}\left(\mathbb{R}^{n}\right) \times \cdots \times L^{p_{m}, \lambda_{m}}\left(\mathbb{R}^{n}\right)$ to $W L^{q, \mu}\left(\mathbb{R}^{n}\right)$.

Moreover, similar estimates hold for $M_{\Omega, \alpha}$.

Proof From Lemma 3.2, we only need to show the boundedness of $T_{\Omega, \alpha}$.

First, we choose $t$ to satisfy $(n-\mu) / q=(n-\lambda) / t$, then we get

$$
1 / t=(n-\mu) / q(n-\lambda)=1 / p-\alpha /(n-\lambda)<1 / p-\alpha / n=1 / q
$$

Then Hölder's inequality implies $L^{t, \lambda}\left(\mathbb{R}^{n}\right) \subset L^{q, \mu}\left(\mathbb{R}^{n}\right)$ and $W L^{t, \lambda}\left(\mathbb{R}^{n}\right) \subset W L^{q, \mu}\left(\mathbb{R}^{n}\right)$. In fact, there exists a constant $C>0$ such that

$$
\left\|T_{\Omega, \alpha} \mathbf{f}\right\|_{L^{q, u}} \leq C\left\|T_{\Omega, \alpha} \mathbf{f}\right\|_{L^{t, \lambda}}
$$

and

$$
\left\|T_{\Omega, \alpha} \mathbf{f}\right\|_{W L}{ }_{W, u} \leq C\left\|T_{\Omega, \alpha} \mathbf{f}\right\|_{W L}{ }_{W, \lambda}
$$


Then, by Theorem 1.1, we have

$$
\left\|T_{\Omega, \alpha} \mathbf{f}\right\|_{L^{q, u}} \leq C\left\|T_{\Omega, \alpha} \mathbf{f}\right\|_{L^{t, \mu}} \leq C \prod_{j=1}^{m}\left\|f_{j}\right\|_{L^{p_{j}, \lambda_{j}}} \quad \text { for } p>s^{\prime}
$$

and

$$
\left\|T_{\Omega, \alpha} \mathbf{f}\right\|_{W L^{q, u}} \leq C\left\|T_{\Omega, \alpha} \mathbf{f}\right\|_{W L^{t, \mu}} \leq C \prod_{j=1}^{m}\left\|f_{j}\right\|_{L^{p_{j}, \lambda_{j}}} \quad \text { for } p=s^{\prime}
$$

Thus, the proof of Corollary 3.1 is completed.

As an another application, by Hölder's inequality, we obtain an Olsen's inequality as in the following corollary, which is a multi-version of the results in considered by Olsen in [12] in the study of the Schrödinger equation with perturbed potentials $W$.

Corollary 3.2 Let $\alpha, \Omega, s, p_{j}, \lambda, p$, and $\lambda$ be as in Theorem 1.1 and let $W \in L^{(n-\lambda) / \alpha, \lambda}$. If $p>s^{\prime}$ and $1 / p-1 / q=\alpha /(n-\lambda)$, then there exists a positive constant $C$ such that

$$
\left\|W \cdot T_{\Omega, \alpha} \mathbf{f}\right\|_{L^{p, \lambda}\left(\mathbb{R}^{n}\right)} \leq C\|W\|_{L^{(n-\lambda) / \alpha, \lambda}\left(\mathbb{R}^{n}\right)} \prod_{j=1}^{m}\left\|f_{j}\right\|_{L^{p_{j}, \lambda}}\left(\mathbb{R}^{n}\right) .
$$

Moreover, similar estimates hold for $M_{\Omega, \alpha}$.

\subsection{Boundedness on modified Morrey spaces}

This part we will devote to the boundedness on modified Morrey spaces and show the proof Theorem 1.2 and 1.3. With the same arguments on Morrey spaces, we also begin with a requisite Hedberg's type estimates on modified Morrey spaces.

Lemma 3.3 Let $0<\alpha<n, \Omega \in L^{s}\left(\mathbb{S}^{n-1}\right)$ with $1<s \leq \infty$, $p$ be the harmonic mean of $p_{1}, \ldots, p_{m}>1,0 \leq \lambda<n-\alpha p, s^{\prime} \leq p<n / \alpha$ and satisfy (1.1), then there exists a positive constant $C$ such that

$$
\left|T_{\Omega, \alpha} \mathbf{f}(x)\right| \leq C\left(M_{\Omega} \mathbf{f}(x)\right)^{p / q} \prod_{j=1}^{m}\left\|f_{j}\right\|_{\widetilde{L}^{p_{j}, \lambda_{j}}}^{1-p / q} .
$$

Proof For any $\delta>0$, we do the same decomoposition of $T_{\Omega, \alpha}$ as in the proof of Lemma 3.1, then we only need to estimate $F_{\sigma}(x, \delta)$. We also choose the same $\sigma$ during the proof of Lemma 3.1, then we get

$$
(n-\sigma) / s^{\prime}-n / p \leq(n-\sigma) / s^{\prime}-(n-\lambda) / p<0 .
$$

If $p>s^{\prime}$, by the Hölder inequality and the fact (3.4), we obtain

$$
\begin{aligned}
F_{\sigma}(x, \delta) & \leq \sum_{i=0}^{\infty}\left(2^{i} \delta\right)^{-\sigma / s^{\prime}}\left(\int_{|y|<2^{i+1} \delta} \prod_{j=1}^{m}\left|f_{j}\left(x-\theta_{j} y\right)\right|^{s^{\prime}} d y\right)^{1 / s^{\prime}} \\
& \leq C \sum_{i=0}^{\infty}\left(2^{i} \delta\right)^{(n-\sigma) / s^{\prime}-n / p}\left(\int_{|y|<2^{i+1} \delta} \prod_{j=1}^{m}\left|f_{j}\left(x-\theta_{j} y\right)\right|^{p} d y\right)^{1 / p}
\end{aligned}
$$




$$
\begin{aligned}
& \leq C \sum_{i=0}^{\infty}\left(2^{i} \delta\right)^{(n-\sigma) / s^{\prime}-n / p}\left[2^{i+1} \delta\right]_{1}^{\lambda / p}\left(\frac{1}{\left[2^{i+1} \delta\right]_{1}^{\lambda}} \int_{|y|<2^{i+1} \delta} \prod_{j=1}^{m}\left|f_{j}\left(x-\theta_{j} y\right)\right|^{p} d y\right)^{1 / p} \\
& \leq C \sum_{i=0}^{\infty}\left(2^{i} \delta\right)^{(n-\sigma) / s^{\prime}-n / p}\left[2^{i+1} \delta\right]_{1}^{\lambda / p} \prod_{j=1}^{m}\left(\frac{1}{\left[2^{i+1} \delta\right]_{1}^{\lambda_{j}}} \int_{|y|<2^{i+1} \delta}\left|f_{j}\left(x-\theta_{j} y\right)\right|^{p_{j}} d y\right)^{1 / p_{j}} \\
& \leq C \sum_{i=0}^{\infty}\left(2^{i} \delta\right)^{(n-\sigma) / s^{\prime}-n / p}\left[2^{i+1} \delta\right]_{1}^{\lambda / p} \prod_{j=1}^{m}\left\|f_{j}\right\|_{\tilde{L}^{p_{j}, \lambda_{j}}} \\
& =C \prod_{j=1}^{m}\left\|f_{j}\right\|_{\tilde{L}^{p_{j}, \lambda_{j}}} \sum_{i=0}^{\infty}\left(2^{i} \delta\right)^{(n-\sigma) / s^{\prime}-n / p}\left[2^{i+1} \delta\right]_{1}^{\lambda / p} \\
& \leq C \prod_{j=1}^{m}\left\|f_{j}\right\|_{\tilde{L}^{p_{j}, \lambda_{j}}} \begin{cases}\sum_{i=0}^{\infty}\left(2^{i} \delta\right)^{(n-\sigma) / s^{\prime}-n / p}, & \text { if } \delta \geq 1 / 2, \\
\sum_{i=0}^{\left[\log _{2} \frac{1}{2 \delta}\right]}\left(2^{i} \delta\right)^{(n-\sigma) / s^{\prime}-(n-\lambda) / p} & \\
\quad+\sum_{i=\left[\log _{2} \frac{1}{2 \delta}\right]+1}^{\infty}\left(2^{i} \delta\right)^{(n-\sigma) / s^{\prime}-n / p}, & \text { if } 0<\delta<1 / 2\end{cases} \\
& \leq C \prod_{j=1}^{m}\left\|f_{j}\right\|_{\tilde{L}^{p_{j}, \lambda_{j}}} \begin{cases}\delta^{(n-\sigma) / s^{\prime}-n / p}, & \text { if } \delta \geq 1 / 2, \\
\delta^{(n-\sigma) / s^{\prime}-(n-\lambda) / p}+\delta^{(n-\sigma) / s^{\prime}-n / p}, & \text { if } 0<\delta<1 / 2\end{cases} \\
& \leq C \prod_{j=1}^{m}\left\|f_{j}\right\|_{\tilde{L}^{p_{j}, \lambda_{j}}} \begin{cases}\delta^{(n-\sigma) / s^{\prime}-n / p}, & \text { if } \delta \geq 1 / 2, \\
\delta^{(n-\sigma) / s^{\prime}-(n-\lambda) / p}, & \text { if } 0<\delta<1 / 2\end{cases} \\
& \leq C \delta^{(n-\sigma) / s^{\prime}-n / p}[2 \delta]_{1}^{\lambda / p} \prod_{j=1}^{m}\left\|f_{j}\right\|_{\tilde{L}^{p_{j}, \lambda_{j}}} .
\end{aligned}
$$

If $p=s^{\prime}$, using the Hölder inequality and the fact $0 \leq \lambda<\sigma$, we get

$$
\begin{aligned}
F_{\sigma}(x, \delta) & \leq \sum_{i=0}^{\infty}\left(2^{i} \delta\right)^{-\sigma / s^{\prime}}\left(\int_{|y|<2^{i+1} \delta} \prod_{j=1}^{m}\left|f_{j}\left(x-\theta_{j} y\right)\right|^{s^{\prime}} d y\right)^{1 / s^{\prime}} \\
& \leq C \sum_{i=0}^{\infty}\left(2^{i} \delta\right)^{-\sigma / s^{\prime}}\left[2^{i+1} \delta\right]_{1}^{\lambda / s^{\prime}}\left(\frac{1}{\left[2^{i+1} \delta\right]_{1}^{\lambda}} \int_{|y|<2^{i+1} \delta} \prod_{j=1}^{m}\left|f_{j}\left(x-\theta_{j} y\right)\right|^{s^{\prime}} d y\right)^{1 / s^{\prime}} \\
& \leq C \sum_{i=0}^{\infty}\left(2^{i} \delta\right)^{-\sigma / s^{\prime}}\left[2^{i+1} \delta\right]_{1}^{\lambda / s^{\prime}} \prod_{j=1}^{m}\left(\frac{1}{\left[2^{i+1} \delta\right]_{1}^{\lambda_{j}}} \int_{|y|<2^{i+1} \delta}\left|f_{j}\left(x-\theta_{j} y\right)\right|^{p_{j}} d y\right)^{1 / p_{j}} \\
& \leq C \prod_{j=1}^{m}\left\|f_{j}\right\|_{\tilde{L}^{p_{j}, \lambda_{j}}} \sum_{i=0}^{\infty}\left(2^{i} \delta\right)^{-\sigma / s^{\prime}}\left[2^{i+1} \delta\right]_{1}^{\lambda / s^{\prime}} \\
& \leq C \prod_{j=1}^{m}\left\|f_{j}\right\|_{\tilde{L}^{p_{j}, \lambda_{j}}}\left\{\begin{array}{l}
\sum_{i=0}^{\infty}\left(2^{i} \delta\right)^{-\sigma / s^{\prime}}, \\
\sum_{i=0}^{\left[\log _{2} \frac{1}{2 \delta}\right]}\left(2^{i} \delta\right)^{(\lambda-\sigma) / s^{\prime}} \\
+\sum_{i=\left[\log _{2} \frac{1}{2 \delta}\right]_{+1}}^{\infty}\left(2^{i} \delta\right)^{-\sigma / s^{\prime}}, \quad \text { if } 0<\delta<1 / 2
\end{array}\right. \\
& \leq C \prod_{j=1}^{m}\left\|f_{j}\right\|_{\tilde{L}_{p_{j}, \lambda_{j}}}\left\{\begin{array}{l}
\delta^{-\sigma / s^{\prime}}, \\
\delta^{(\lambda-\sigma) / s^{\prime}}+\delta^{-\sigma / s^{\prime}}, \quad \text { if } 0<\delta<1 / 2,
\end{array}\right. \\
& \leq C \prod_{j=1}^{m}\left\|f_{j}\right\|_{\tilde{L}_{L_{j}, \lambda}, \lambda_{j}}\left\{\begin{array}{l}
\delta^{-\sigma / s^{\prime}}, \quad \text { if } \delta \geq 1 / 2, \\
\delta^{(\lambda-\sigma) / s^{\prime}}, \quad \text { if } 0<\delta<1 / 2
\end{array}\right.
\end{aligned}
$$




$$
\begin{aligned}
& \leq C \delta^{-\sigma / s^{\prime}}[2 \delta]_{1}^{\lambda / p} \prod_{j=1}^{m}\left\|f_{j}\right\|_{\tilde{L}^{p_{j}, \lambda_{j}}} \\
& \leq C \delta^{(n-\sigma) / s^{\prime}-n / p}[2 \delta]_{1}^{\lambda / p} \prod_{j=1}^{m}\left\|f_{j}\right\|_{\tilde{L}^{p_{j}, \lambda_{j}}} .
\end{aligned}
$$

Then, combining with the estimates $E_{\sigma}(\delta) \leq C \delta^{\alpha-(n-\sigma) / s^{\prime}}$, we have

$$
|S(x, \delta)| \leq C \delta^{\alpha-n / p}[2 \delta]_{1}^{\lambda / p} \prod_{j=1}^{m}\left\|f_{j}\right\|_{\tilde{L}^{p_{j}, \lambda_{j}}}, \quad \text { for every } p \geq s^{\prime} .
$$

Thus

$$
\begin{aligned}
\left|T_{\Omega, \alpha} \mathbf{f}(x)\right| \leq & C\left(\delta^{\alpha} M_{\Omega} \mathbf{f}(x)+\delta^{\alpha-n / p}[2 \delta]_{1}^{\lambda / p} \prod_{j=1}^{m}\left\|f_{j}\right\|_{\tilde{L}^{p_{j}, \lambda_{j}}}\right) \\
\leq & C \min \left\{\delta^{\alpha} M_{\Omega} \mathbf{f}(x)+\delta^{\alpha-n / p} \prod_{j=1}^{m}\left\|f_{j}\right\|_{\tilde{L}^{p_{j}, \lambda_{j}}},\right. \\
& \left.\delta^{\alpha} M_{\Omega} \mathbf{f}(x)+\delta^{\alpha-(n-\lambda) / p} \prod_{j=1}^{m}\left\|f_{j}\right\|_{\tilde{L}^{p_{j}, \lambda_{j}}}\right\} .
\end{aligned}
$$

Minimizing with respect to $\delta$, at

$$
\delta=\left[\left(M_{\Omega} \mathbf{f}(x)\right)^{-1} \prod_{j=1}^{m}\left\|f_{j}\right\|_{\widetilde{L}^{p_{j}, \lambda_{j}}}\right]^{p / n}
$$

and

$$
\delta=\left[\left(M_{\Omega} \mathbf{f}(x)\right)^{-1} \prod_{j=1}^{m}\left\|f_{j}\right\|_{\widetilde{L}^{p_{j}, \lambda_{j}}}\right]^{p /(n-\lambda)}
$$

we have

$$
\begin{aligned}
\left|T_{\Omega, \alpha} \mathbf{f}(x)\right| & \leq C \min \left\{\left(\frac{M_{\Omega} \mathbf{f}(x)}{\prod_{j=1}^{m}\left\|f_{j}\right\|_{\tilde{L}^{p_{j}, \lambda_{j}}}}\right)^{1-\frac{p \alpha}{n}},\left(\frac{M_{\Omega} \mathbf{f}(x)}{\prod_{j=1}^{m}\left\|f_{j}\right\|_{\tilde{L}^{p_{j}, \lambda_{j}}}}\right)^{1-\frac{p \alpha}{n-\lambda}}\right\} \prod_{j=1}^{m}\left\|f_{j}\right\|_{\widetilde{L}^{p_{j}, \lambda_{j}}} \\
& \leq C\left(M_{\Omega} \mathbf{f}(x)\right)^{p / q} \prod_{j=1}^{m}\left\|f_{j}\right\|_{\widetilde{L}^{p_{j}, \lambda_{j}}}^{1-p / q} .
\end{aligned}
$$

This is the conclusion of Lemma 3.3 .

Now we give the proof of Theorem 1.2.

Proof of Theorem 1.2 Similarly to the proof of sufficiency in Theorem 1.1, by the boundedness of $M_{\Omega}$ in Theorem 2.4, we will get the sufficiency. Now, we give only the proof of necessity. 
Let $[\epsilon]_{1,+}=\max \{1, \epsilon\}$, by (3.3), for $\mathbf{f}_{\epsilon}(x)$ with $\epsilon>0$, we get

$$
\begin{aligned}
\left\|T_{\Omega, \alpha} \mathbf{f}_{\epsilon}\right\|_{\widetilde{L}^{q, \lambda}}= & \epsilon^{-\alpha} \sup _{x \in \mathbb{R}^{n}, t>0}\left(\frac{1}{[t]_{1}^{\lambda}} \int_{B(x, t)}\left|T_{\Omega, \alpha} \mathbf{f}(\epsilon y)\right|^{q} d y\right)^{1 / q} \\
= & \epsilon^{-\alpha-n / q} \sup _{x \in \mathbb{R}^{n}, t>0}\left(\frac{1}{[t]_{1}^{\lambda}} \int_{B(\epsilon x, \epsilon t)}\left|T_{\Omega, \alpha} \mathbf{f}(y)\right|^{q} d y\right)^{1 / q} \\
= & \epsilon^{-\alpha-n / q} \sup _{t>0}\left(\frac{[\epsilon t]_{1}}{[t]_{1}}\right)^{\lambda / q} \\
& \times \sup _{x \in \mathbb{R}^{n}, t>0}\left(\frac{1}{[\epsilon t]_{1}^{\lambda}} \int_{B(\epsilon x, \epsilon t)}\left|T_{\Omega, \alpha} \mathbf{f}(y)\right|^{q} d y\right)^{1 / q} \\
= & \epsilon^{-\alpha-n / q}[\epsilon]_{1,+}^{\frac{\lambda}{q}}\left\|T_{\Omega, \alpha} \mathbf{f}\right\|_{\tilde{L}^{q, \lambda}}
\end{aligned}
$$

and

$$
\begin{aligned}
\left\|T_{\Omega, \alpha} \mathbf{f}_{\epsilon}\right\|_{W \widetilde{L}} q, \lambda= & \sup _{r>0} r \sup _{x \in \mathbb{R}^{n}, t>0}\left(\frac{1}{[t]_{1}^{\lambda}} \int_{\left\{y \in B(x, t):\left|T_{\Omega, \alpha} \mathbf{f}_{\epsilon}(y)\right|>r\right\}} d y\right)^{\frac{1}{q}} \\
= & \sup _{r>0} r \sup _{x \in \mathbb{R}^{n}, t>0}\left(\frac{1}{[t]_{1}^{\lambda}} \int_{\left\{y \in B(x, t):\left|T_{\Omega, \alpha} \mathbf{f}(\epsilon y)\right|>r \epsilon^{\alpha}\right\}} d y\right)^{\frac{1}{q}} \\
= & \epsilon^{-n / q} \sup _{r>0} r \sup _{x \in \mathbb{R}^{n}, t>0}\left(\frac{1}{[t]_{1}^{\lambda}} \int_{\left\{y \in B(\epsilon x, \epsilon t):\left|T_{\Omega, \alpha} \mathbf{f}(y)\right|>r \epsilon^{\alpha}\right\}} d y\right)^{\frac{1}{q}} \\
= & \epsilon^{-\alpha-n / q} \sup _{t>0}\left(\frac{[\epsilon t]_{1}}{[t]_{1}}\right)^{\lambda / q} \\
& \times \sup _{r>0} r \epsilon^{\alpha} \sup _{x \in \mathbb{R}^{n}, t>0}\left(\frac{1}{[\epsilon t]_{1}^{\lambda}}\left|\left\{y \in B(\epsilon x, \epsilon t):\left|T_{\Omega, \alpha} \mathbf{f}(y)\right|>r \epsilon^{\alpha}\right\}\right|\right)^{\frac{1}{q}} \\
= & \epsilon^{-\alpha-n / q}[\epsilon]_{1,+}^{\frac{\lambda}{q}}\left\|T_{\Omega, \alpha} \mathbf{f}\right\|_{W \tilde{L}^{q, \lambda} .}
\end{aligned}
$$

(i) Assume that $T_{\Omega, \alpha}$ is bounded from $\widetilde{L}^{p_{1}, \lambda_{1}} \times \cdots \times \widetilde{L}^{p_{m}, \lambda_{m}}$ to $\widetilde{L}^{q, \lambda}$, we get

$$
\begin{aligned}
\left\|T_{\Omega, \alpha} \mathbf{f}\right\|_{\tilde{L}^{q, \lambda}}= & \epsilon^{\alpha+n / q}[\epsilon]_{1,+}^{-\frac{\lambda}{q}}\left\|T_{\Omega, \alpha} \mathbf{f}_{\epsilon}\right\|_{\tilde{L}^{q, \lambda}} \\
\leq & C \epsilon^{\alpha+n / q}[\epsilon]_{1,+}^{-\frac{\lambda}{q}} \prod_{j=1}^{m}\left\|f_{j}(\epsilon \cdot)\right\|_{\widetilde{L}^{p_{j}, \lambda_{j}}} \\
= & C \epsilon^{\alpha+n / q}[\epsilon]_{1,+}^{-\frac{\lambda}{q}} \prod_{j=1}^{m} \sup _{x \in \mathbb{R}^{n}, t>0}\left(\frac{1}{[t]_{1}^{\lambda_{j}}} \int_{B(x, t)}\left|f_{j}(\epsilon y)\right|^{p_{j}} d y\right)^{1 / p_{j}} \\
= & C \epsilon^{\alpha+n / q}[\epsilon]_{1,+}^{-\frac{\lambda}{q}} \prod_{j=1}^{m} \epsilon^{-n / p_{j}} \sup _{x \in \mathbb{R}^{n}, t>0}\left(\frac{1}{[t]_{1}^{\lambda_{j}}} \int_{B(\epsilon x, \epsilon t)}\left|f_{j}(y)\right|^{p_{j}} d y\right)^{1 / p_{j}} \\
\leq & C \epsilon^{\alpha+n / q}[\epsilon]_{1,+}^{-\frac{\lambda}{q}} \prod_{j=1}^{m} \epsilon^{-n / p_{j}} \sup _{t>0}\left(\frac{[\epsilon t]_{1}}{[t]_{1}}\right)^{\lambda_{j} / p_{j}} \\
& \times \sup _{x \in \mathbb{R}^{n}, t>0}\left(\frac{1}{[\epsilon t]^{\lambda_{j}}} \int_{B(\epsilon x, \epsilon t)}\left|f_{j}(y)\right|^{p_{j}} d y\right)^{1 / p_{j}}
\end{aligned}
$$




$$
\begin{aligned}
& \leq C \epsilon^{\alpha+n / q-n / p}[\epsilon]_{1,+}^{-\frac{\lambda}{q}}[\epsilon]_{1,+}^{\frac{\lambda}{p}} \prod_{j=1}^{m}\left\|f_{j}\right\|_{\widetilde{L}^{p_{j}, \lambda_{j}}} \\
& \leq C \epsilon^{\alpha+n / q-n / p}[\epsilon]_{1,+}^{\frac{\lambda}{p}-\frac{\lambda}{q}} \prod_{j=1}^{m}\left\|f_{j}\right\|_{\widetilde{L}^{p_{j}, \lambda_{j}}},
\end{aligned}
$$

where $C$ is independent of $\epsilon$.

When $1 / p<1 / q+\alpha / n$, then for all $\mathbf{f} \in \widetilde{L}^{p_{1}, \lambda_{1}} \times \cdots \times \widetilde{L}^{p_{m}, \lambda_{m}}$, we have $\left\|T_{\Omega, \alpha} \mathbf{f}\right\|_{\widetilde{L}^{q, \lambda}}=0$ as $\epsilon \rightarrow 0$.

When $1 / p>1 / q+\alpha /(n-\lambda)$, then for all $\mathbf{f} \in \widetilde{L}^{p_{1}, \lambda_{1}} \times \cdots \times \widetilde{L}^{p_{m}, \lambda_{m}}$, we have $\left\|T_{\Omega, \alpha} \mathbf{f}\right\|_{\widetilde{L}^{q, \lambda}}=0$ as $\epsilon \rightarrow \infty$.

Therefore we get $\alpha / n \leq 1 / p-1 / q \leq \alpha /(n-\lambda)$.

(ii) Assume that $T_{\Omega, \alpha}$ is bounded from $\widetilde{L}^{p_{1}, \lambda_{1}} \times \cdots \times \widetilde{L}^{p_{m}, \lambda_{m}}$ to $W \widetilde{L}^{q, \lambda}$, we have

$$
\begin{aligned}
\left\|T_{\Omega, \alpha} \mathbf{f}\right\|_{W \widetilde{L} q, \lambda} & =\epsilon^{\alpha+n / q}[\epsilon]_{1,+}^{\frac{-\lambda}{q}}\left\|T_{\Omega, \alpha} \mathbf{f}_{\epsilon}\right\|_{W \widetilde{L} q, \lambda} \leq C \epsilon^{\alpha+n / q}[\epsilon]_{1,+}^{-\frac{\lambda}{q}} \prod_{j=1}^{m}\left\|f_{j}(\epsilon \cdot)\right\|_{\widetilde{L}^{p_{j}, \lambda_{j}}} \\
& \leq C \epsilon^{\alpha+n / q-n / p}[\epsilon]_{1,+}^{\frac{\lambda}{\bar{p}}-\frac{\lambda}{q}} \prod_{j=1}^{m}\left\|f_{j}\right\|_{\widetilde{L}^{p_{j}, \lambda_{j}}}
\end{aligned}
$$

where $C$ is independent of $\epsilon$.

When $1 / p<1 / q+\alpha / n$, then for all $\mathbf{f} \in \widetilde{L}^{p_{1}, \lambda} \times \cdots \times \widetilde{L}^{p_{m}, \lambda}$, we have $\left\|T_{\Omega, \alpha} \mathbf{f}\right\|_{W \widetilde{L}^{q, \lambda}}=0$ as $\epsilon \rightarrow 0$

When $1 / p>1 / q+\alpha /(n-\lambda)$, then for all $\mathbf{f} \in \widetilde{L}^{p_{1}, \lambda} \times \cdots \times \widetilde{L}^{p_{m}, \lambda}$, we have $\left\|T_{\Omega, \alpha} \mathbf{f}\right\|_{W \widetilde{L}^{q, \lambda}}=0$ as $\epsilon \rightarrow \infty$.

Consequently, we get $\alpha / n \leq 1 / p-1 / q \leq \alpha /(n-\lambda)$.

Next, we prove conclusions (i) and (ii) hold for $M_{\Omega, \alpha}$. By the same arguments as above we get the necessity part and the sufficiency part follows from the conclusion of $T_{\Omega, \alpha}$ and Lemma [9].

This completes the proof of Theorem 1.2.

Finally we show the proof of Theorem 1.3.

Proof of Theorem 1.3 By the Hölder inequality, we have

$$
\begin{aligned}
M_{\Omega, \alpha} \mathbf{f}(x) & =\sup _{r>0} \frac{1}{r^{n-\alpha}} \int_{|y|<r}|\Omega(y)| \prod_{j=1}^{m}\left|f_{j}\left(x-\theta_{j} y\right)\right| d y \\
& \leq C \sup _{r>0} \frac{1}{r^{n-\alpha}}\left(\int_{|y|<r}|\Omega(y)|^{p^{\prime}} d y\right)^{1 / p^{\prime}}\left(\int_{|y|<r} \prod_{j=1}^{m}\left|f_{j}\left(x-\theta_{j} y\right)\right|^{p} d y\right)^{1 / p} \\
& \leq C \sup _{r>0} \frac{1}{r^{n-\alpha}}\left(\int_{|y|<r}|\Omega(y)|^{p^{\prime}} d y\right)^{1 / p^{\prime}} \prod_{j=1}^{m}\left(\int_{|y|<r}\left|f_{j}\left(x-\theta_{j} y\right)\right|^{p_{j}} d y\right)^{1 / p_{j}} \\
& \leq C \sup _{r>0} r^{\alpha-n / p}\left(\frac{1}{r^{n}} \int_{|y|<r}|\Omega(y)|^{s} d y\right)^{1 / s} \prod_{j=1}^{m}\left(\int_{|y|<r}\left|f_{j}\left(x-\theta_{j} y\right)\right|^{p_{j}} d y\right)^{1 / p_{j}} \\
& \leq C \sup _{r>0} r^{\alpha-n / p} \prod_{j=1}^{m}\left(\int_{|y|<r}\left|f_{j}\left(x-\theta_{j} y\right)\right|^{p_{j}} d y\right)^{1 / p_{j}} \cdot
\end{aligned}
$$


(i) If $p=(n-\lambda) / \alpha \geq s^{\prime}$, by the fact (1.1), we obtain

$$
\begin{aligned}
M_{\Omega, \alpha} \mathbf{f}(x) & \leq C \sup _{r>0} r^{\alpha-(n-\lambda) / p} \prod_{j=1}^{m}\left(\frac{1}{r^{\lambda_{j}}} \int_{|y|<r}\left|f_{j}\left(x-\theta_{j} y\right)\right|^{p_{j}} d y\right)^{1 / p_{j}} \\
& =C \prod_{j=1}^{m}\left\|f_{j}\right\|_{L^{p_{j}, \lambda_{j}}} .
\end{aligned}
$$

(ii) If $(n-\lambda) / \alpha \leq p \leq n / \alpha$, using the fact (1.1), we get

$$
\begin{aligned}
M_{\Omega, \alpha} \mathbf{f}(x) & \leq C \sup _{r>0} r^{\alpha-n / p}[r]_{1}^{\lambda / p} \prod_{j=1}^{m}\left(\frac{1}{[r]_{1}^{\lambda_{j}}} \int_{|y|<r}\left|f_{j}\left(x-\theta_{j} y\right)\right|^{p_{j}} d y\right)^{1 / p_{j}} \\
& \leq C \sup _{r>0} r^{\alpha-n / p}[r]_{1}^{\lambda / p} \prod_{j=1}^{m}\left\|f_{j}\right\|_{\widetilde{L}^{p_{j}, \lambda_{j}}} \\
& \leq C \prod_{j=1}^{m}\left\|f_{j}\right\|_{\widetilde{L}^{p_{j}, \lambda_{j}}} \max \left\{\sup _{0<r<1} r^{\alpha-\frac{n-\lambda}{p}}, \sup _{r \geq 1} r^{\alpha-n / p}\right\} \\
& =C \prod_{j=1}^{m}\left\|f_{j}\right\|_{\widetilde{L}^{p_{j}, \lambda_{j}}} .
\end{aligned}
$$

Therefore, we complete the proof of Theorem 1.3.

Finally, we would like to remark that our theorems generalize the relevant results in [13-15].

\section{Competing interests}

The authors declare that they have no competing interests.

\section{Authors' contributions}

All authors read and approved the final manuscript.

\section{Author details}

${ }^{1}$ Zhejiang Pharmaceutical College, Ningbo, Zhejiang 315100, P.R. China. ${ }^{2}$ School of Mathematics and Information Science, Henan Polytechnic University, Jiaozuo, Henan 454000, P.R. China. ${ }^{3}$ Faculty of Science, Zhejiang University of Science \& Technology, Hangzhou, Zhejiang 310023, P.R. China. ${ }^{4}$ School of Science, Ningbo University of Technology, Ningbo, Zhejiang 315211, P.R. China. ${ }^{5}$ School of Statistics and Management, Shanghai University of Finance and Economics, Shanghai, 200433, P.R. China.

\section{Acknowledgements}

The research of the first author was supported by the Soft Science Foundation of Ningbo City (No. 2015A10001). The second author was supported by the National Natural Science Foundation of China (No. 11401175 and No. 11501169). The third author was supported by the National Natural Science Foundation of China (No. 11171306). Thanks are also given to the anonymous referees for careful reading and suggestions.

Received: 18 March 2015 Accepted: 6 December 2015 Published online: 04 February 2016

\section{References}

1. Lu, S, Ding, Y, Yan, D: Singular Integrals and Related Topics. World Scientific, Singapore (2006)

2. Stein, EM: Harmonic Analysis: Real-Variable Methods, Orthogonality, and Oscillatory Integrals. Princeton University Press, Princeton (1993)

3. Guliyev, V, Shukurov, P: On the boundedness of the fractional maximal operator, Riesz potential and their commutators in generalized Morrey spaces. Oper. Theory, Adv. Appl. 229, 175-199 (2013)

4. Peetre, J: On the theory of $L^{p, \lambda}$. J. Funct. Anal. 4, 71-87 (1969)

5. Adams, DR: A note on Riesz potentials. Duke Math. J. 42, 765-778 (1975)

6. Guliyev, V, Hasnov, J, Zeren, Y: Necesary and sufficient conditions for the boundedness of the Riesz potential in modified Morrey spaces. J. Math. Inequal. 5, 491-506 (2011) 
7. Grafakos, L: On multilinear fractional integrals. Stud. Math. 102, $49-56$ (1992)

8. Gunawan, H, Sihwaningrum, I: Multilinear maximal functions and fractional integrals on generalized Morrey spaces. http://personal.fmipa.itb.ac.id/hgunawan/files/2007/11/multilinear-maximal-functions-n-fractional-integralsver-3.pdf (2014). Accessed 26 Dec 2014

9. Ding, Y, Lu, S: The $L^{p_{1}} \times L^{p_{2}} \times \cdots \times L^{p_{k}}$ boundedness for some rough operators. J. Math. Anal. Appl. 203, 166-186 (1996)

10. Shi, $Y$, Tao, $X$ : Multilinear Riesz potential operators on Herz-type spaces and generalized Morrey spaces. Hokkaido Math. J. 38, 635-662 (2009)

11. Nakai, E: Hardy-Littlewood maximal operator, singular integral operators and the Riesz potentials on generalized Morrey spaces. Math. Nachr. 166, 95-103 (1994)

12. Olsen, PA: Fractional integration, Morrey spaces and a Schrödinger equation. Commun. Partial Differ. Equ. 20 2005-2055 (1995)

13. Kenig, CE, Stein, EM: Multilinear estimates and fractional integration. Math. Res. Lett. 6, 1-15 (1999)

14. Shi, Y, Tao, X: The boundedness of sublinear operators on Morrey-Herz spaces over the homogeneous type space. Anal. Math. 39, 69-85 (2013)

15. Shi, $Y$, Tao, X: Some multi-sublinear operators on generalized Morrey spaces with non-doubling measures. J. Korean Math. Soc. 49, 907-925 (2012)

Submit your manuscript to a SpringerOpen ${ }^{\circ}$ journal and benefit from:

- Convenient online submission

- Rigorous peer review

- Immediate publication on acceptance

- Open access: articles freely available online

- High visibility within the field

- Retaining the copyright to your article 